\title{
Variations saisonnières des helminthes gastro-intestinaux chez la chèvre du plateau central du Burkina Faso
}

\author{
A.M.G. Belem ${ }^{1} *$ A. Kaboré ${ }^{2}$ R. Bessin ${ }^{3}$
}

Mots-clés

Caprin - Helminthe - Nematoda Cestoda - Trematoda - Burkina Faso.

\section{Résumé}

Des nécropsies pendant une année de 60 chèvres ont permis d'identifier les parasites de la cavité abdominale, du foie et du tube digestif. Au total, 10 nématodes, 4 cestodes et 2 trématodes ont été identifiés. Cysticercus tenuicollis a été trouvé dans la cavité péritonéale, Dicrocoelium hospes dans le foie, et Gongylonema verrucosum et des paramphistomes dans l'ensemble réseau-rumenfeuillet. Dans la caillette, il y avait Haemonchus contortus et Trichostrongylus colubriformis. L'intestin grêle abritait T. colubriformis, T. vitrinus, Strongyloides papillosus, Gaigeria pachyscelis, Avitellina centripunctata, Stilesia globipunctata et Moniezia expansa. Quant au gros intestin, il hébergeait Oesophagostomum columbianum, Skrjabinema ovis, Trichuris ovis et Trichuris globulosa. Les plus prévalents de ces parasites ont été T. colubriformis (98,3 p. 100), Oesophagostomum columbianum (88,3 p. 100), les larves L4 (85 p. 100), puis les adultes (65 p. 100) d'Haemonchus contortus, T. vitrinus (41,7 p. 100), Avitellina centripunctata (30 p. 100) et Cysticercus tenuicollis (30 p. 100). L'abondance moyenne des nématodes a globalement été faible à l'exception de celle des larves $\mathrm{d}^{\prime} H$. contortus dans la caillette et des adultes de $T$. colubriformis dans I'intestin grêle à la fin de la saison pluvieuse, et pendant la période sèche et froide. Cette période serait idéale pour l'application du deuxième déparasitage stratégique annuel des chèvres de la région.
1. IDR, université polytechnique de Bobo-Dioulasso, 01 BP 3770, Ouagadougou 01, Burkina Faso

Tél. : +22650357295; ; e-mail : amg.belem@ fasonet.bf amgbelem@hotmail.com

2. Inera, DPA-Kamboinsé, 01 BP 476, Ouagadougou 01, Burkina Faso

3. UA, IBAR, PACE, BP 30786, 00100 Nairobi, Kenya

* Auteur pour la correspondance

\section{INTRODUCTION}

Au Burkina Faso, les petits ruminants procurent une source importante de protéines animales dans l'alimentation en particulier des populations rurales. De plus, ils sont souvent vendus pour les besoins financiers des éleveurs et utilisés dans les cérémonies coutumières. Malgré cela, peu d'études ont été réalisées sur la pathologie parasitaire des petits ruminants, notamment des chèvres, alors qu'il est connu que les parasites gastro-intestinaux entrainent des pertes économiques importantes pour les éleveurs $(1,4,10,15)$.

Avant d'étudier l'impact pathologique des parasites gastro-intestinaux sur la productivité des ruminants, il apparaît indispensable de donner les précisions suivantes sur la nature exacte des parasites incriminés, leur importance numérique, leur localisation anatomique sur les hôtes et l'impact des saisons sur leur biologie. L'amélioration de la productivité des petits ruminants, tant recherchée au Burkina Faso, passe par la connaissance de ces données épizootiologiques de base non encore élucidées. Pour contribuer à cela, cette 
étude a eu pour objectif spécifique l'identification et l'énumération de tous les helminthes gastro-intestinaux dans leurs sites anatomiques de prédilection et à leurs stades spécifiques de développement, l'évaluation de l'impact des saisons sur la faune parasitaire et la proposition de périodes stratégiques adaptées de déparasitage.

\section{MATERIEL ET METHODES}

\section{Zone et animaux de l'étude}

De février 2002 à janvier 2003, cinq chèvres de race locale Mossi ont été abattues mensuellement pour l'étude. Tous ces animaux provenaient du plateau central du Burkina Faso, localisé dans les zones climatiques sahélienne et nord-soudanienne (7). Les cinq chèvres étaient choisies chaque mois au hasard parmi un grand lot d'animaux conduits par des éleveurs ou des commerçants au marché à bétail de Pouytenga, localisé dans la région de l'étude. Etant conduites au marché pour être vendues, les chèvres étaient globalement d'âge assez semblable tout au long de l'année. Les élevages d'origine des chèvres étudiées étaient des élevages traditionnels mixtes de bovins, ovins et caprins où les animaux n'étaient pas traités avec des anthelminthiques et ne subissaient aucun regroupement particulier des mises bas. La pluviométrie annuelle dans la zone varie entre 500 à $900 \mathrm{~mm}$. On y trouve également deux grandes saisons climatiques : la saison pluvieuse de juin à octobre et la saison sèche de novembre à mai. Cette dernière peut être subdivisée en deux plus petites saisons : la saison sèche froide, allant d'environ novembre à février avec des extrêmes de températures de 8 à $15^{\circ} \mathrm{C}$ la nuit et de 30 à $37^{\circ} \mathrm{C}$ le jour, et la saison sèche chaude, allant d'environ mars à mai avec des extrêmes de températures de 27 à $35^{\circ} \mathrm{C}$ la nuit et de 31 à $45^{\circ} \mathrm{C}$ le jour.

Avant l'abattage à l'abattoir frigorifique d'Ouagadougou, chaque chèvre était soigneusement examinée et les informations suivantes étaient notées : village d'origine, sexe, poids, âge estimé par l'observation de la table dentaire, pathologie visible, aspect général et date d'abattage.

\section{Méthode d'échantillonnage à l'abattoir}

Après la saignée de l'animal, l'abdomen a été ouvert et la cavité péritonéale examinée pour la recherche de vésicules du ténia $C y s$ ticercus tenuicollis. Ensuite, l'ensemble du tube digestif a été retiré de la cavité abdominale. A l'aide de fil, des doubles ligatures ont été réalisées autour de la caillette à la jonction iléo-cæcale et une seule ligature au bout du rectum avant de séparer avec un couteau les différentes portions du tube digestif que sont l'ensemble réseaurumen-feuillet, la caillette, l'intestin grêle et le gros intestin. Ces opérations ont été réalisées le plus rapidement possible pour limiter les transferts éventuels des contenus des différentes portions du tube digestif après la mort de l'animal. Enfin, les graisses et les mésentères au niveau des viscères ont été dégarnis au maximum. Les différentes portions du tube digestif et le foie ont été individuellement placés dans un seau en plastique identifié et envoyé au laboratoire pour l'analyse.

\section{Récupération, identification et énumération des parasites}

Les méthodes de récupération, d'identification et d'énumération de nématodes digestifs décrites par Couvillion et coll. (5), et Belem et coll. $(2,3)$ ont été suivies avec de légères modifications. Pour les parasites de la cavité péritonéale, de l'ensemble réseau-rumenfeuillet et du foie, une observation qualitative a été réalisée, suivie de la récolte pour identification de quelques parasites dans du formol à 10 p. $100(\mathrm{v} / \mathrm{v})$. Pour ce faire, la cavité péritonéale a été simplement examinée, alors que l'ensemble réseau-rumen-feuillet a été ouvert avec un couteau et son contenu soigneusement fouillé pour la recherche de paramphistomes ou autres parasites. Sur le foie, la vésicule et les canaux biliaires ont été ouverts, puis lavés sur un tamis à ouvertures de $38 \mu \mathrm{m}$. Ce qui est resté du tamisage a été observé à la loupe binoculaire pour la recherche de douves ou autres parasites qui ont été préservés dans le formol à 10 p. 100.

La caillette a été ouverte longitudinalement et son contenu versé dans un seau en plastique gradué. Dans le même seau gradué et sous un fin jet d'eau de robinet, la muqueuse de la caillette a été soigneusement rincée. L'ensemble des liquides diluant le contenu de la caillette dans le seau a été porté à $5 \mathrm{l}$, puis a été correctement homogénéisé avant que ne soient prélevées deux aliquotes de $500 \mathrm{ml}$ (environ 10 p. 100 du volume total), chacune à l'aide d'un gobelet. Chacune des deux aliquotes a été clarifiée sous des jets d'eau de robinet à travers le tamis, réduite à un plus petit volume et conservée à l'aide du formol à 10 p. 100 dans un flacon approprié et étiqueté jusqu'au moment de l'observation à la loupe binoculaire pour la récupération des parasites. La première et la deuxième aliquote ont été successivement étiquetées aliquotes originale et double. Entre deux échantillons, tout le matériel utilisé a été soigneusement rincé à l'eau de robinet et au savon.

Après son lavage, la caillette a été placée dans un récipient, trempée dans de l'eau et incubée à la température ambiante du laboratoire pendant 16 à $18 \mathrm{~h}$. Pendant cette période les récipients ont été couverts pour éloigner les mouches. Après l'incubation, la muqueuse de la caillette a été légèrement grattée avec un couteau sous un jet d'eau de robinet. Tous ces liquides issus de l'incubation et du nettoyage de la caillette ont été portés à 51 dans un seau gradué et comme précédemment deux aliquotes ont été prélevées et préservées chacune avec du formol dans un flacon étiqueté.

L'intestin grêle et le gros intestin ont été traités séparément mais de la même manière que la caillette. Ils ont été ouverts longitudinalement et leur contenu a été versé dans un seau gradué. Les muqueuses ont été rincées trois fois de suite sous un jet d'eau de robinet. L'ensemble des liquides obtenus a été porté à 51 et deux aliquotes ont été prélevées et traitées comme mentionné ci-dessus. Entre deux échantillons traités, tout le matériel utilisé a été soigneusement rincé à l'eau et au savon. Les intestins n'ont pas été incubés dans l'eau de robinet comme la caillette.

Toutes les aliquotes réalisées dans le formol ont été gardées à la température ambiante du laboratoire jusqu'à leur utilisation. L'aliquote originale a été utilisée pour la récolte des parasites. Avant son utilisation pour la récolte de parasites, l'aliquote a été à nouveau passée à travers le tamis de $38 \mu \mathrm{m}$ sous un jet d'eau de robinet pour diminuer la forte odeur de formol lors de la récolte des parasites sous la loupe binoculaire. Les parasites de la caillette récoltés de l'incubation ont été traités séparément de ceux récoltés du contenu. Versée en petites quantités dans une boîte de Petri marquée par des grilles, l'aliquote a été entièrement observée sous une loupe binoculaire. Tout élément parasitaire ou ressemblant à un parasite a été récupéré de nouveau dans du formol à 10 p. 100. Ensuite, les larves et les adultes des parasites ont été transférés sur des lames de microscope avec des gouttes de lactophénol et recouverts par des lamelles jusqu'à leur identification et énumération. Ces lames bien identifiées ont été gardées dans des boîtes de rangement à l'abri de la poussière. A chaque fois qu'un très grand nombre de parasites étaient observés dans une aliquote, des sous-aliquotes étaient réalisées et utilisées pour la récolte de parasites.

L'identification des parasites gastro-intestinaux a été faite à l'aide d'un microscope équipé d'un oculaire micrométrique et d'objectifs calibrés à l'aide d'un micromètre objet $(2,12)$. Si possible, chaque parasite a été identifié selon le sexe, le stade de développement, 


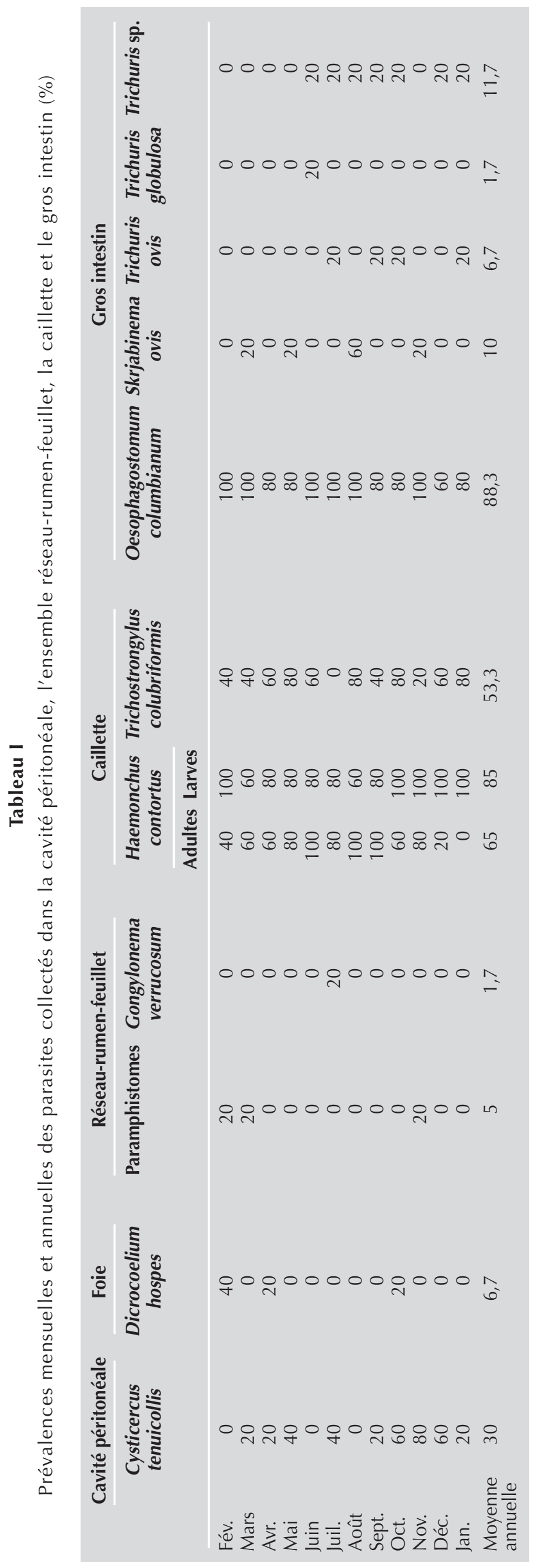

le genre et l'espèce. Le nombre total d'un type de parasite pour une localisation donnée a été calculé en fonction du coefficient de dilution de l'aliquote utilisée. Pour la caillette, a été réalisée la somme des effectifs du parasite dans le contenu et les liquides d'incubation pour le calcul du nombre total du parasite.

\section{Analyses statistiques}

Alors que les taux de prévalence ou les pourcentages ont été analysés par le test du Chi deux, les nombres moyens de parasites ont été comparés par l'analyse des variances (Anova) suivie de la méthode de séparation des moyennes de Student-Newman-Keuls (SNK), au seuil de 5 p. 100. Toutes les analyses statistiques ont été réalisées grâce au logiciel SAS (14).

\section{RESULTATS}

\section{Nature des helminthes gastro-intestinaux}

Les différents parasites collectés dans la cavité péritonéale, le foie et le tube digestif des chèvres examinées ont été représentés par 10 nématodes, 4 cestodes ( 3 formes adultes et 1 cysticerque) et 2 trématodes (tableaux I et II). Pendant l'année de l'étude, les parasites les plus fréquemment rencontrés ont été les suivants: Trichostrongylus colubriformis (98,3 p. 100), Oesophagostomum columbianum (88,3 p. 100), les larves L4 et les adultes d'Haemonchus contortus (respectivement 85 et 65 p. 100), Trichostrongylus vitrinus (41,7 p. 100), Avitellina centripunctata (30 p. 100), et Cysticercus tenuicollis (30 p. 100).

Les tableaux I et II montrent la localisation anatomique de chaque type de parasite récolté. Il est intéressant de noter la présence de T. colubriformis aussi bien dans l'intestin grêle que dans la caillette avec toutefois une plus grande importance dans le premier site (tableaux I à IV). Les larves L4 ont été identifiées selon l'espèce dans la caillette et seulement selon le genre dans l'intestin grêle où elles étaient moins importantes (tableaux I à IV). Elles ont été représentées dans la caillette par $H$. contortus et dans l'intestin grêle par Trichostrongylus sp. avec des prévalences annuelles respectives de 85 et 16,7 p. 100 .

\section{Variations saisonnières des prévalences parasitaires}

\section{Parasites de la cavité péritonéale, du foie et de l'ensemble réseau-rumen-feuillet}

C. tenuicollis, la larve de Taenia hydatigena, a été trouvé dans la cavité péritonéale durant presque toute l'année avec toutefois de plus grandes fréquences en fin de saison pluvieuse, d'octobre à décembre. Le trématode Dicrocoelium hospes et des paramphistomes ont été présents respectivement dans le foie et le réseaurumen-feuillet en saison sèche de février à avril et en fin de saison pluvieuse en octobre et novembre. Le nématode Gongylonema verrucosum a été observé dans le réseau-rumen-feuillet seulement en juillet (20 p. 100) pendant la saison pluvieuse (tableau I).

\section{Parasites de la caillette}

La caillette a été principalement habitée par les larves et les adultes d' $H$. contortus et secondairement par les adultes de $T$. colubriformis (tableau I). Les prévalences mensuelles des larves et des adultes d'H. contortus ont été similaires et élevées la plupart du temps à l'exception de la période allant de la fin de la saison pluvieuse à la petite saison sèche et froide (octobre à février) où les prévalences des larves ont été très significativement élevées $\left(\chi^{2}=27,6\right.$; d.d.l. $=11 ; \mathrm{p}=0,004)$ et celles des adultes significativement faibles $\left(\chi^{2}=24,8 ;\right.$ d.d.l. $\left.=11 ; p=0,01\right)$. A l'exception du mois de juillet, 


\section{Tableau II}

Prévalences mensuelles et annuelles des parasites collectés dans l'intestin grêle (\%)

\begin{tabular}{|c|c|c|c|c|c|c|c|c|c|}
\hline & $\begin{array}{l}\text { richostrongylus } \\
\text { colubriformis }\end{array}$ & T. vitrinus & $\begin{array}{l}\text { T. sp. } \\
\text { larves }\end{array}$ & $\begin{array}{c}\text { Strongyloides } \\
\text { papillosus }\end{array}$ & $\begin{array}{c}\text { Gaigeria } \\
\text { pachyscelis }\end{array}$ & $\begin{array}{c}\text { Avitellina } \\
\text { centripunctata }\end{array}$ & $\begin{array}{c}\text { Stilesia } \\
\text { globipunctata }\end{array}$ & $\begin{array}{c}\text { Moniezia } \\
\text { expansa }\end{array}$ & $\begin{array}{l}\text { Total } \\
\text { ténias }\end{array}$ \\
\hline Fév. & 100 & 0 & 20 & 0 & 0 & 20 & 0 & 0 & 20 \\
\hline Mars & 100 & 60 & 20 & 0 & 20 & 0 & 0 & 0 & 0 \\
\hline Avr. & 100 & 80 & 0 & 20 & 0 & 20 & 0 & 20 & 20 \\
\hline Mai & 100 & 60 & 20 & 40 & 20 & 20 & 0 & 0 & 20 \\
\hline Juin & 100 & 20 & 0 & 20 & 0 & 40 & 20 & 0 & 40 \\
\hline Juil. & 100 & 20 & 40 & 0 & 0 & 60 & 0 & 0 & 60 \\
\hline Août & 80 & 20 & 0 & 20 & 20 & 40 & 0 & 0 & 40 \\
\hline Sept. & 100 & 60 & 20 & 0 & 0 & 20 & 0 & 0 & 20 \\
\hline Oct. & 100 & 20 & 20 & 0 & 0 & 20 & 0 & 0 & 20 \\
\hline Nov. & 100 & 80 & 20 & 0 & 40 & 40 & 20 & 0 & 60 \\
\hline Déc. & 100 & 60 & 0 & 0 & 20 & 60 & 0 & 0 & 60 \\
\hline Jan. & 100 & 20 & 40 & 0 & 20 & 20 & 0 & 0 & 20 \\
\hline $\begin{array}{l}\text { Moyenne } \\
\text { annuelle }\end{array}$ & 98,3 & 41,7 & 16,7 & 8,3 & 11,7 & 30 & 3,3 & 1,7 & 31,7 \\
\hline
\end{tabular}

les adultes du parasite intestinal T. colubriformis ont été observés toute l'année dans la caillette mais à des prévalences mensuelles faibles de 20 à 80 p. 100.

\section{Parasites de l'intestin grêle}

Les prévalences mensuelles de T. colubriformis ont été beaucoup plus importantes dans l'intestin grêle (80 à 100 p. 100) que dans la caillette (0 à 80 p. 100). La presque totalité des 60 chèvres autopsiées au cours de l'étude en possédait dans les intestins grêles. T. vitrinus n'a été observé que dans les intestins grêles, et cela à des prévalences mensuelles inférieures à celles de T. colubriformis (0 à 80 p. 100). Les larves des Trichostrongylus qui n'ont pu être identifiées selon l'espèce ont été relativement moins fréquentes que les adultes avec une prévalence annuelle de 16,7 p. 100 (tableau II). Strongyloides papillosus a été trouvé dans l'intestin grêle des chèvres à des prévalences mensuelles de 0 à 40 p. 100, essentiellement en saison pluvieuse d'avril à août. Gaigeria pachyscelis a été observée dans les intestins grêles à des prévalences mensuelles allant de 0 à 40 p. 100. Trois différentes espèces de cestodes ont été trouvées dans les intestins grêles: Moniezia expansa, Stilesia globipunctata et Avitellina centripunctata à des prévalences annuelles respectives de 1,7, 3,3 et 30 p. 100. Ensemble, la prévalence annuelle des cestodes adultes a été de 31,7 p. 100.

\section{Parasites du gros intestin}

Quatre espèces de parasites ont été trouvées dans le gros intestin des chèvres : Oesophagostomum columbianum (88,3 p. 100), Trichuris ovis (6,7 p. 100), T. globulosa (1,7 p. 100) et Skrjabinema ovis (10 p. 100) (tableau I). Les femelles des Trichuris qui n'ont pu être identifiées selon l'espèce ont contribué à élever la prévalence annuelle du genre à 11,7 p. 100, repartie en saison pluvieuse (juin à octobre) et en saison sèche et froide (décembre à janvier).

\section{Variations saisonnières des abondances moyennes des nématodes}

\section{Nématodes de la caillette}

Les abondances moyennes mensuelles de $H$. contortus ont été la plupart du temps plus élevées pour les larves que pour les adultes, sauf en juin et en août, en pleine saison pluvieuse (tableau III).
Pour les larves d' $H$. contortus, l'abondance moyenne mensuelle la plus élevée a été observée en décembre avec 6676 larves par chèvre (l'abondance maximale de larves étant de 15 230, observée en janvier) ; l'abondance moyenne la moins élevée a été observée en août avec 147 larves par chèvre (l'abondance minimale de larves étant de 10, observée en avril et mai). L'abondance moyenne mensuelle des adultes d' $H$. contortus la plus élevée a été observée en septembre avec 946 adultes par chèvre (le maximum étant de 2330 parasites adultes); l'abondance moyenne la moins élevée a été de 0 adulte par chèvre en janvier. Les comparaisons statistiques des abondances moyennes mensuelles des larves d' $H$. contortus par la méthode d'Anova complétée par la méthode SNK de séparation des moyennes ont montré que le mois de décembre était très significativement différent des autres mois $(\mathrm{p}<0,001)$. Les comparaisons statistiques des abondances moyennes mensuelles des adultes d' $H$. contortus par la méthode d'Anova ont montré qu'il existait des différences très significatives entre les mois $(\mathrm{p}<0,001)$.

Pour les adultes de T. colubriformis récoltés dans la caillette, les abondances moyennes mensuelles observées ont varié de 0 parasite par chèvre en juillet à 272 parasites en janvier.

\section{Nématodes des intestins}

L'abondance parasitaire mensuelle de T. colubriformis chez les chèvres a été de loin plus importante dans les intestins grêles que dans les caillettes. De même, les adultes de ce parasite ont été beaucoup plus nombreux que ses larves tous les mois de l'année (tableaux III et IV). L'abondance parasitaire mensuelle de T. colubriformis a varié de 396 à 3736 parasites par mois et par animal pour les adultes et de 0 à 200 pour les larves. Le mois de novembre a été le mois avec l'abondance moyenne la plus élevée pour les adultes de T. colubriformis, le maximum observé étant de 8400 . L'abondance parasitaire moyenne de T. colubriformis a été statistiquement plus élevée $(\mathrm{p}<0,001)$ aux mois de novembre, janvier et février de la saison sèche et froide qu'aux autres mois de l'année. Pour T. vitrinus, les abondances moyennes mensuelles ont été plus faibles, variant de 0 à 80 parasites par chèvre examinée. Strongyloides papillosus, observé en fin de saison sèche et début de saison pluvieuse, a montré des abondances moyennes mensuelles relativement faibles, allant de 0 à 40 parasites par animal. G. pachyscelis, récolté surtout en saison sèche et froide, a présenté également 


\section{Tableau III}

Moyennes \pm écarts-types (et extrêmes) des effectifs de nématodes récoltés dans la caillette et le gros intestin

\begin{tabular}{|c|c|c|c|c|c|c|c|c|}
\hline & \multicolumn{3}{|c|}{ Caillette } & \multicolumn{5}{|c|}{ Gros intestin } \\
\hline & \multicolumn{2}{|c|}{$\begin{array}{l}\text { Haemonchus } \\
\text { contortus }\end{array}$} & \multirow[t]{2}{*}{$\begin{array}{l}\text { Trichostrongylus } \\
\text { colubriformis }\end{array}$} & \multirow[t]{2}{*}{$\begin{array}{l}\text { Oesophagostomum } \\
\text { columbianum }\end{array}$} & \multirow[t]{2}{*}{$\begin{array}{c}\text { Skrjabinema } \\
\text { ovis }\end{array}$} & \multirow[t]{2}{*}{$\begin{array}{l}\text { Trichuris } \\
\text { ovis }\end{array}$} & \multirow[t]{2}{*}{$\begin{array}{l}\text { Trichuris } \\
\text { globulosa }\end{array}$} & \multirow[t]{2}{*}{ Trichuris sp } \\
\hline & Adultes & Larves & & & & & & \\
\hline Fév. & $\begin{array}{l}100 \pm 28 \\
(80-120)\end{array}$ & $\begin{array}{r}1966 \pm 1147 \\
(840-3870)\end{array}$ & $\begin{array}{l}115 \pm 78 \\
(60-170)\end{array}$ & $\begin{array}{l}96 \pm 82 \\
(20-200)\end{array}$ & 0 & 0 & 0 & 0 \\
\hline Mars & $\begin{array}{l}97 \pm 60 \\
(40-160)\end{array}$ & $\begin{array}{c}493 \pm 392 \\
(40-720)\end{array}$ & $\begin{array}{l}70 \pm 71 \\
(20-120)\end{array}$ & $\begin{array}{l}80 \pm 42 \\
(20-120)\end{array}$ & $\begin{array}{l}620 \pm 0 \\
(620-620)\end{array}$ & 0 & 0 & 0 \\
\hline Avr. & $\begin{array}{c}183 \pm 193 \\
(30-400)\end{array}$ & $\begin{array}{r}1482 \pm 1672 \\
(10-3000)\end{array}$ & $\begin{array}{l}37 \pm 15 \\
(20-50)\end{array}$ & $\begin{array}{l}110 \pm 89 \\
(40-240)\end{array}$ & 0 & 0 & 0 & 0 \\
\hline Mai & $\begin{array}{l}162 \pm 59 \\
(80-220)\end{array}$ & $\begin{array}{l}420 \pm 709 \\
(10-1480)\end{array}$ & $\begin{array}{l}72 \pm 70 \\
(20-170)\end{array}$ & $\begin{array}{c}90 \pm 50 \\
(40-160)\end{array}$ & $\begin{array}{l}600 \pm 0 \\
(600-600)\end{array}$ & 0 & 0 & 0 \\
\hline Juin & $\begin{array}{l}892 \pm 711 \\
(200-2080)\end{array}$ & $\begin{array}{l}432 \pm 673 \\
(40-1440)\end{array}$ & $\begin{array}{l}147 \pm 46 \\
(120-200)\end{array}$ & $\begin{array}{l}216 \pm 308 \\
(40-760)\end{array}$ & 0 & 0 & $\begin{array}{l}40 \pm 0 \\
(40-40)\end{array}$ & $\begin{array}{l}80 \pm 0 \\
(80-80)\end{array}$ \\
\hline Juil. & $\begin{array}{l}540 \pm 52 \\
(480-600)\end{array}$ & $\begin{array}{l}632 \pm 468 \\
(50-1160)\end{array}$ & 0 & $\begin{array}{c}54 \pm 46 \\
(10-120)\end{array}$ & 0 & $\begin{array}{l}20 \pm 0 \\
(20-20)\end{array}$ & 0 & $\begin{array}{l}20 \pm 0 \\
(20-20)\end{array}$ \\
\hline Août & $\begin{array}{c}882 \pm 868 \\
(240-2330)\end{array}$ & $\begin{array}{c}147 \pm 122 \\
(40-280)\end{array}$ & $\begin{array}{c}150 \pm 124 \\
(40-320)\end{array}$ & $\begin{array}{c}86 \pm 131 \\
(10-320)\end{array}$ & $\begin{array}{l}427 \pm 636 \\
(20-1160)\end{array}$ & 0 & 0 & $\begin{array}{l}20 \pm 0 \\
(20-20)\end{array}$ \\
\hline Sept. & $\begin{array}{c}946 \pm 651 \\
(200-1600)\end{array}$ & $\begin{array}{r}1460 \pm 2506 \\
(20-5210)\end{array}$ & $\begin{array}{c}60 \pm 28 \\
(40-80)\end{array}$ & $\begin{array}{l}110 \pm 68 \\
(40-200)\end{array}$ & 0 & $\begin{array}{l}40 \pm 0 \\
(40-40)\end{array}$ & 0 & $\begin{array}{l}40 \pm 0 \\
(40-40)\end{array}$ \\
\hline Oct. & $\begin{array}{c}280 \pm 160 \\
(120-440)\end{array}$ & $\begin{array}{l}714 \pm 778 \\
(50-2030)\end{array}$ & $\begin{array}{c}210 \pm 136 \\
(40-320)\end{array}$ & $\begin{array}{c}52 \pm 34 \\
(10-80)\end{array}$ & 0 & $\begin{array}{l}10 \pm 0 \\
(10-10)\end{array}$ & 0 & $\begin{array}{l}10 \pm 0 \\
(10-10)\end{array}$ \\
\hline Nov. & $\begin{array}{c}410 \pm 376 \\
(120-960)\end{array}$ & $\begin{array}{l}4522 \pm 4498 \\
(200-10160)\end{array}$ & $\begin{array}{l}80 \pm 0 \\
(80-80)\end{array}$ & $\begin{array}{l}80 \pm 40 \\
(40-120)\end{array}$ & $\begin{array}{l}200 \pm 0 \\
(200-200)\end{array}$ & 0 & 0 & 0 \\
\hline Déc. & $\begin{array}{l}640 \pm 0 \\
(640-640)\end{array}$ & $\begin{array}{c}6676 \pm 5677 \\
(300-13350)\end{array}$ & $\begin{array}{c}137 \pm 40 \\
\quad(90-160)\end{array}$ & $\begin{array}{c}93 \pm 46 \\
(40-120)\end{array}$ & 0 & 0 & 0 & $\begin{array}{c}80 \pm 0 \\
(80-80)\end{array}$ \\
\hline Jan. & 0 & $\begin{array}{c}3990 \pm 6372 \\
(130-15230)\end{array}$ & $\begin{array}{r}272 \pm 379 \\
(50-840)\end{array}$ & $\begin{array}{l}100 \pm 52 \\
(40-160)\end{array}$ & 0 & $\begin{array}{l}40 \pm 0 \\
(40-40)\end{array}$ & 0 & $\begin{array}{l}40 \pm 0 \\
(40-40)\end{array}$ \\
\hline
\end{tabular}

des abondances moyennes mensuelles relativement faibles allant de 0 à 60 parasites par chèvre.

Comparativement aux autres compartiments du tube digestif, le gros intestin a été le moins chargé en parasites. O. columbianum, le plus fréquent des parasites à ce niveau, a présenté l'abondance moyenne mensuelle la plus élevée en juin avec une moyenne de 216 parasites par chèvre (l'abondance maximale observée étant de 760 parasites). Skrjabinema ovis a été plus rare qu'O. columbianum mais a présenté des abondances moyennes plus élevées, particulièrement en mars avec 620 parasites par chèvre. Les parasites du genre Trichuris ont été les moins nombreux dans le gros intestin, avec des abondances moyennes mensuelles variant de 0 à 80 parasites par chèvre.

\section{Impact de l'âge, du poids et du sexe de l'animal sur l'abondance parasitaire}

Le nombre de dents adultes (DA) de l'animal examiné a été considéré pour la détermination de l'âge, permettant ainsi de constituer cinq catégories de chèvres par rapport à l'âge : les chèvres de 0 , $2,4,6$ et 8 DA. Aucune différence significative $(p>0,05)$ n'a pu être trouvée entre les abondances moyennes mensuelles des parasites pour tous les groupes d'âge constitués. L'abondance parasitaire des animaux regroupés en trois catégories différentes en fonction du poids de la carcasse (moins de $9 \mathrm{~kg}$, entre 10 et $12 \mathrm{~kg}$ et audelà de $13 \mathrm{~kg}$ ) n'a également pas révélé de différence significative
( $p>0,05)$ entre les groupes. Pareillement, aucune différence significative n'a été trouvée $(\mathrm{p}>0,05)$ entre les chèvres mâles et femelles par rapport à l'abondance parasitaire mensuelle.

\section{DISCUSSION}

Les méthodes de recherche des parasites utilisées dans cette étude ont permis d'identifier sur la chèvre du plateau central du Burkina Faso 16 espèces différentes de parasites dans la cavité péritonéale, le foie et le tube digestif. Il y avait 10 espèces de nématodes (Gongylonema verrucosum, Haemonchus contortus, Trichostrongylus colubriformis, T. vitrinus, Strongyloides papillosus, Gaigeria pachyscelis, Oesophagostomum columbianum, Skrjabinema ovis, Trichuris ovis et Trichuris globulosa), 4 espèces de cestodes (Avitellina centripunctata, Moniezia expansa, Stilesia globipunctata et Cysticercus tenuicollis) et 2 types de trématodes (Dicrocoelium hospes et des paramphistomes). Ces méthodes n'ont pas permis de collecter d'autres types de parasites tels que les protozoaires.

Les parasites trouvés sur les chèvres du plateau central du Burkina Faso ont déjà été décrits sur ces animaux dans d'autres pays de l'Afrique de l'Ouest (1, 4, 9, 11, 12, 13). Néanmoins, il est important de noter que des parasites tels que Trichostrongylus axei, Cooperia sp. et Oesophagostomum radiatum trouvés ailleurs $(1,11,13)$ n'ont pas été observés dans cette étude. 


\section{Tableau IV}

Moyennes \pm écart-types (et extrêmes) des effectifs de nématodes récoltés dans l'intestin grêle

\begin{tabular}{|c|c|c|c|c|c|}
\hline & $\begin{array}{c}\text { Trichostrongylus } \\
\text { colubriformis }\end{array}$ & $\begin{array}{c}T . \\
\text { vitrinus }\end{array}$ & $\begin{array}{l}\text { Trichostrongylus sp. } \\
\text { larves }\end{array}$ & $\begin{array}{c}\text { Strongyloides } \\
\text { papillosus }\end{array}$ & $\begin{array}{l}\text { Gaigeria } \\
\text { pachyscelis }\end{array}$ \\
\hline Fév. & $\begin{array}{r}3024 \pm 2097 \\
(760-5320)\end{array}$ & 0 & $\begin{array}{l}80 \pm 0 \\
(80-80)\end{array}$ & 0 & 0 \\
\hline Mars & $\begin{array}{c}396 \pm 653 \\
(40-1560)\end{array}$ & $\begin{array}{c}27 \pm 11 \\
(20-40)\end{array}$ & $\begin{array}{l}200 \pm 0 \\
(200-200)\end{array}$ & 0 & $\begin{array}{l}60 \pm 0 \\
(60-60)\end{array}$ \\
\hline Avr. & $\begin{array}{l}1040 \pm 872 \\
(520-2560)\end{array}$ & $\begin{array}{l}55 \pm 30 \\
(40-100)\end{array}$ & 0 & $\begin{array}{c}40 \pm 0 \\
(40-40)\end{array}$ & 0 \\
\hline Mai & $\begin{array}{r}1340 \pm 1681 \\
(220-4320)\end{array}$ & $\begin{array}{l}73 \pm 50 \\
(20-120)\end{array}$ & $\begin{array}{l}10 \pm 0 \\
(10-10)\end{array}$ & $\begin{array}{l}25 \pm 7 \\
(20-30)\end{array}$ & $\begin{array}{l}10 \pm 0 \\
(10-10)\end{array}$ \\
\hline Juin & $\begin{array}{r}1592 \pm 1437 \\
(80-3920)\end{array}$ & $\begin{array}{l}80 \pm 0 \\
(80-80)\end{array}$ & 0 & $\begin{array}{c}40 \pm 0 \\
(40-40)\end{array}$ & 0 \\
\hline Juil. & $\begin{array}{l}572 \pm 496 \\
(140-1360)\end{array}$ & $\begin{array}{c}40 \pm 0 \\
(40-40)\end{array}$ & $\begin{array}{c}30 \pm 14 \\
(20-40)\end{array}$ & 0 & 0 \\
\hline Août & $\begin{array}{l}500 \pm 539 \\
(80-1240)\end{array}$ & $\begin{array}{l}40 \pm 0 \\
(40-40)\end{array}$ & 0 & $\begin{array}{c}40 \pm 0 \\
(40-40)\end{array}$ & $\begin{array}{l}40 \pm 0 \\
(40-40)\end{array}$ \\
\hline Sept. & $\begin{array}{c}1768 \pm 458 \\
(1280-2400)\end{array}$ & $\begin{array}{c}67 \pm 23 \\
(40-80)\end{array}$ & $\begin{array}{c}80 \pm 0 \\
(80-80)\end{array}$ & 0 & 0 \\
\hline Oct. & $\begin{array}{c}1176 \pm 832 \\
(280-2280)\end{array}$ & $\begin{array}{l}40 \pm 0 \\
(40-40)\end{array}$ & $\begin{array}{l}120 \pm 0 \\
(120-120)\end{array}$ & 0 & 0 \\
\hline Nov. & $\begin{array}{c}3736 \pm 2959 \\
(1360-8400)\end{array}$ & $\begin{array}{c}70 \pm 20 \\
(40-80)\end{array}$ & $\begin{array}{l}200 \pm 0 \\
(200-200)\end{array}$ & 0 & $\begin{array}{c}60 \pm 28 \\
(40-80)\end{array}$ \\
\hline Déc. & $\begin{array}{c}1024 \pm 460 \\
(400-1600)\end{array}$ & $\begin{array}{l}67 \pm 46 \\
(40-120)\end{array}$ & 0 & $\begin{array}{c}0 \\
(40-40)\end{array}$ & $40 \pm 0$ \\
\hline Jan. & $\begin{array}{r}2824 \pm 2484 \\
(40-6040)\end{array}$ & $\begin{array}{l}80 \pm 0 \\
(80-80)\end{array}$ & $\begin{array}{c}60 \pm 28 \\
(40-80)\end{array}$ & 0 & $\begin{array}{c}40 \pm 0 \\
(40-40)\end{array}$ \\
\hline
\end{tabular}

Dans le travail mené, les parasites suivants ont été les plus prévalents sur les chèvres étudiées : T. colubriformis (98,3 p. 100), $O$. columbianum $(88,3)$ et $H$. contortus $(85$ p. 100). A cause de leurs larves, les parasites d' $H$. contortus ont été cependant les plus nombreux, pouvant atteindre un maximum de 15230 larves sur une chèvre. L'infestation par des parasites gastro-intestinaux dans la zone d'étude peut être considérée très commune car aucun animal examiné n'a été trouvé indemne de parasites. De plus, les polyinfestations semblaient plutôt être la règle, ce qui devrait susciter un intérêt particulier pour l'étude de l'impact pathologique des associations parasitaires sur les chèvres.

L'analyse des prévalences et des abondances mensuelles laisse percevoir que la période d'explosion parasitaire sur les chèvres du plateau central du Burkina Faso se situerait de la fin de la saison pluvieuse à janvier. Même si la saison pluvieuse est la période climatique la plus favorable pour la phase externe de développement des parasites dans la nature et la période par excellence d'infestation des animaux, leurs prévalences et leurs effectifs seraient maximaux de la fin de la saison pluvieuse (septembre) à la période sèche et froide (décembre et janvier). $H$. contortus connaîtrait apparemment un cycle de développement très complexe impliquant la possibilité de traverser la dure période sèche de l'année dans les deux états larvaires L4 et adultes. L'arrêt de développement chez ce parasite a déjà été décrit par certains auteurs $(11,15)$ et pourrait être dû à des réactions immunologiques de leurs hôtes ou bien être sous l'influence du climat. De février à août, la prévalence d' $H$. contortus aux stades de larves et d'adultes augmenterait chez les animaux. Alors que d'octobre à janvier, tout en existant toujours à l'état adulte, ils seraient plus nombreux à l'état larvaire.
Les résultats de cette étude montrent alors qu' $H$. contortus se préserverait en tant qu'espèce biologique dans la nature en utilisant cette stratégie d'augmentation de sa prévalence sur les animaux et d'inhibition de son développement au stade larvaire L4 au cours, en particulier, des périodes climatiques très défavorables de l'année. Les autres espèces de nématodes observées ne présenteraient qu'une stratégie unique au cours de cette même période, celle de rester au stade adulte.

O. columbianum, collecté tous les mois, apparaît comme un parasite constant des chèvres de la région d'étude. Il serait judicieux de poursuivre cette étude sur des animaux plus jeunes pour évaluer l'importance des parasites tels que les coccidies et Strongyloides sp. Egalement, il serait très utile de connaître l'impact de ces parasites sur l'état sanitaire des chèvres au Burkina Faso.

\section{CONCLUSION}

Cette étude, menée au cours d'une année entière, a permis de confirmer l'existence réelle de parasites dans le tractus digestif des chèvres du plateau central du Burkina Faso, de préciser la nature exacte des espèces et d'évaluer leur importance numérique. Au niveau des responsables de l'élevage des chèvres, une plus grande attention devrait être accordée à la présence d'H. contortus dans la caillette, de $T$. colubriformis et des cestodes dans l'intestin grêle et d'O. columbianum dans le gros intestin. Les traitements anthelminthiques stratégiques habituellement effectués dans la région en fin de saison sèche et de saison pluvieuse devront être poursuivis, en effectuant toutefois le deuxième traitement de fin de saison sèche 
le plus tard possible, de préférence au cours de la saison sèche et froide. De plus, les résultats confirment la nécessité de traiter tous les animaux du troupeau et d'utiliser des produits à très large spectre pour lutter contre les polyinfestations constatées.

\section{Remerciements}

Les auteurs sont très reconnaissants à la Fondation internationale pour la science (FIS) pour le soutien financier accordé à la réalisation de cette étude (bourse $n^{\circ} \mathrm{B} / 2358-2$ ). Ils voudraient par ailleurs adresser leurs remerciements à tout le personnel technique et administratif du Laboratoire national d'élevage du ministère des Ressources animales du Burkina Faso qui a bien volontiers aménagé de l'espace pour l'équipe de recherche.

\section{BIBILIOGRAPHIE}

1. ASSOKU R.K.G., 1983. Gastro-intestinal helminth parasites of cattle in Ghana. Bull. Anim. Health Prod. Afr., 31: 223-230.

2. BELEM A.M.G., COUVILLION C.E., SIEFKER C., GRIFFIN R.N., 1993. Evidence for arrested development of abomasal nematodes in whitetailed deer. J. Wild. Dis., 29: 261-265.

3. BELEM A.M.G., OUEDRAOGO O.P., BESSIN R., 2001. Gastrointestinal nematodes and cestodes of cattle in Burkina Faso. Biotechnol. Agron. Soc. Environ., 5: 17-21.

4. CHOLLET J.Y., MARTRENCHAR A., BOUCHEL D., NJOYA A., 1994. Epidémiologie des parasitoses digestives des jeunes bovins dans le NordCameroun. Revue Elev. Méd. vét. Pays trop., 47 : 365-374.

\section{Summary}

Belem A.M.G., Kaboré A., Bessin R. Seasonal Variations of Gastrointestinal Helminths in the Goat of the Central Plateau of Burkina Faso

Necropsies of 60 goats were performed over a year to identify parasites in the abdominal cavity, the liver, and the digestive tube. Overall, 10 nematodes, 4 cestodes, and 2 trematodes were identified. Cysticercus tenuicollis was found in the peritoneal cavity, Dicrocoelium hospes in the liver, and Gongylonema verrucosum and paramphistomes in the reticulumrumen-omasum. In the abomasum, Haemonchus contortus and Trichostrongylus colubriformis were present. In the small intestine were present T. colubriformis, T. vitrinus, Strongyloides papillosus, Gaigeria pachyscelis, Avitellina centripunctata, Stilesia globipunctata, and Moniezia expansa. The large intestine harbored Oesophagostomum columbianum, Skrjabinema ovis, Trichuris ovis, and Trichuris globulosa. The most prevalent parasites were $T$. colubriformis $(98.3 \%), O$. columbianum $(88.3 \%), H$. contortus L4s $(85 \%)$ and adults $(65 \%)$, T. vitrinus $(41.7 \%)$, Avitellina centripunctata (30\%), and Cysticercus tenuicollis $(30 \%)$. The mean nematode burden was globally low with the exception of $H$. contortus L4s in the abomasum and T. colubriformis adults in the small intestine at the end of the rainy season, and during the dry and cold period. This period would be ideal for the second annual strategic deworming of goats in the area.

Keywords: Goat - Helminth - Nematoda - Cestoda Trematoda - Burkina Faso.
5. COUVILLION C.E., CROW C.B., WILLIAM R.D., 1982. An evaluation of hunter-killed white-tailed deer for abomasal parasite count (APC) studies. Proc. annu. Conf. Southeast. Assoc. Fish Wildl. Agencies, 36 427-435.

6. EYSKER M., KOOYMANN F.N.J., 1993. Notes on necropsy and herbage processing techniques for gastro-intestinal nematodes of ruminants. Vet. Parasitol., 46: 205-213.

7. GUINKO S., 1984. Végétation de la Haute Volta. Thèse Doct. Sci., université de Bordeaux III, France, 318 p.

8. HART J.A., 1964. Observations on the dry season strongyle infestations of zebu cattle in Northern Nigeria. Br. vet. J., 120: 87-95.

9. HORAK I.G., 1978. Parasites of domestic and wild animals in South Africa. XI. Helminths in cattle on natural pastures in the northern Transvaal. Onderst. J. vet. Res., 45: 229-234.

10. INERA, 1994. Synthèse des recherches sur la production animale au Burkina Faso. Ouagadougou, Burkina Faso, Inera, 176 p.

11. KAUFMANN J., PFISTER K., 1990. The seasonal epidemiology of gastro-intestinal nematodes in N'Dama cattle in the Gambia. Vet Parasitol., 37: 45-54.

12. PANDEY V.S., CHITATE F., NYANZUNDA T.M., 1993. Epidemiological observations on gastro-intestinal nematodes in communal land cattle from the highveld of Zimbabwe. Vet. Parasitol., 51: 99-106.

13. OUATTARA L., DORCHIES P., 2001. Helminthes gastro-intestinaux des moutons et chèvres en zones sub-humide et sahélienne du Burkina Faso. Revue Méd. vét., 152 : 165-170.

14. SAS INSTITUTE, 1987. SAS user's guide: Statistics, Vers. 6. Cary, NC, USA, SAS Institute, $584 \mathrm{p}$.

15. SOULSBY E.J.L., 1986. Helminths, arthropods and protozoa of domesticated animals, $7^{\text {th }}$ edn. London, UK, Baillière Tindall, $809 \mathrm{p}$.

Reçu le 14.12.2004, accepté le 13.05.2005

\section{Resumen}

Belem A.M.G., Kaboré A., Bessin R. Variaciones estacionarias de los helmintos gastrointestinales en la cabra de la meseta central de Burkina Faso

Durante un año, las necropsias de 60 cabras permitieron la identificación de los parásitos de la cavidad abdominal, del hígado y del tubo digestivo. En total, fueron identificados 10 nemátodos, 4 céstodos y 2 tremátodos. Cysticercus tenuicollis fue encontrado en la cavidad peritoneal, Dicrocoelium hospes en el hígado y Gongylonema verrucosum y los paranfistomos en el conjunto redecilla-rumen-librillo. En el abomaso, había Haemonchus contortus y Trichostrongylus colubriformis. El intestino delgado hospedaba T. colubriformis, T. vitrinus, Strongyloides papillosus, Gaigeria pachyscelis, AviteIlina centripunctata, Stilesia globipunctata y Moniezia expansa. En cuanto al intestino grueso, albergaba Oesophagostomum columbianum, Skrjabinema ovis, Trichuris ovis y Trichuris globulosa. Los parásitos de mayor prevalencia fueron: T. colubriformis (98,3 \%), Oesophagostomum columbianum (88,3 \%), las larvas L4 (85 \%) y los adultos (65\%) de Haemonchus contortus, T. vitrinus (41,7 \%), Avitellina centripunctata (30\%) y Cysticercus tenuicollis (30\%). La abundancia media de los nemátodos fue globalmente baja, con la excepción de la de las larvas de $H$. contortus en el abomaso y de los adultos de $T$. colubriformis en el intestino delgado al final de la estación Iluviosa y durante el periodo seco y frío. Este periodo sería ideal para la aplicación de la segunda desparasitación estratégica anual de las cabras de la región.

Palabras clave: Caprino - Helminto - Nematoda - Cestoda Trematoda - Burkina Faso. 